DOI: $10.17957 / \mathrm{IJAB} / 15.1891$

http://www.fspublishers.org

\title{
Enhanced Growth and Drought Resistance in Seedlings of Acacia tortilis due to Inoculation of Arbuscular Mycorrhiza Fungi and Bacillus subtilis
}

\author{
Abdelmalik M Abdelmalik*, Thobayet S Alshahrani and Abdulaziz A Al-Qarawi \\ Department of Plant production, Faculty of Food and Agriculture Sciences, King Saud University, Saudi Arabia \\ *For correspondence: aadam1@ksu.edu.sa \\ Received 15 October 2021; Accepted 23 November 2021; Published 15 December 2021
}

\begin{abstract}
A shade house experiment was conducted in Saudi Arabia to evaluate the impact of a mixture of three arbuscular mycorrhiza fungi (AMF) namely Funneliformis mosseae, Rhizophagus intraradices and Claroideoglomus etunicatum, a bacterium Bacillus subtilis, and their combinations on the growth and drought resistance potential of Acacia torilis seedlings under moderate and water deficit-stress. Thus, inoculants treatments (AMF, Bacillus subtilis, AMF+Bacillus, and control) and several watering intervals (1, 2, 3 and 4 weeks) were applied. Inoculation of AMF and Bacillus to A. tortilis seedlings found effective in terms of improved seedling growth. AMF and combined inoculation resulted in a larger shoot (shoot fresh and dry weights, seedling height, leaf number, leaf area) and root development (root fresh and dry weights, root length, root surface area, and root volume) as compared to the non-inoculated seedlings. Single inoculants of B. subtilis, showed better improvement in 1- and 2-week watering intervals compared to the control. Inoculated seedlings showed lower proline accumulation than non-inoculated seedlings, and thus improved seedling resistance to water deficit-stress. Mycorrhizal and mixed inoculation enhanced the amount of chlorophyll in the seedling's leaves. Furthermore, seedlings with AMF and co-inoculants showed better drought tolerance even at 3- and 4-week watering intervals. (C) 2021 Friends Science Publishers
\end{abstract}

Keywords: Acacia tortilis; AMF; Bacillus subtilis; Co-inoculation; Deficit-stress

\section{Introduction}

Drought and climate change are great challenges that is being faced by forest ecosystems today (Bhuyan et al. 2017) and prediction of climate change models suggest that drought risk will rise in tropical forests during the next years. Results of different experiments state that drought can cause a reduction in trees development and amplified trees mortality (Richard 2016). Drought can cause significant environmental effects and is likely to increase in many places in the world with climate change (Amanda et al. 2016), particularly in the arid regions, where water is a limiting factor that controls plant growth and survival (Kondoh et al. 2006).

A large number of plants make symbiotic relationship with microorganisms in the soils to overcome the negative impacts of the drought (Wang and Qui 2006; Nadeem et al. 2014). Rhizosphere microorganisms have a decisive role on the growth of plants established under limiting soil environments (Hashem et al. 2019). Microorganism association provides essential resources to the plant, and that in turn will improve the performance of plant to cope with drought (Liddycoat et al. 2009). Different ecophysiological studies have stated that AMF symbiosis is a key factor that assists plants to cope with water stress and increase drought tolerance (Javaid 2009; Rapparini and
Penuelas 2014). Yooyongwech et al. (2013) showed that AMF symbiosis enhanced chlorophyll content in woody trees under water deficit conditions. The inoculation of plant growth promoting bacteria (PGPB) singly or combined with other microorganisms (such as AMF) are widespread and their application is rising in global farming practices (Díaz-Zorita and Fernández-Canigia 2009; Sharf et al. 2021). The combination of AMF fungi and Bacillus subtilis increased the fresh and dry biomass production of aromatic plants (Alam et al. 2011). Hashem et al. (2015) concluded that there is a positive effect of B. subtilis on the growth of inoculated plants. Also, they stated that $B$. subtilis strain caused significant increase in chlorophyll $a$ and $b$ content in leaves of Bassia indica.

Acacia tortilis is one of the widespread tree across the dry-lands of African continent (especially Sudan) and Middle East and has a great role for several groups of pastoral communities (Andersen 2012). The tree is considered an important species of the arid region in many African and Asian countries, where provides building wood, shade, forage, shelter for people and animals, richness the biodiversity, and keep soil fertile, so that it is considered a keystone species (Maarten et al. 2015; Verma 2016).

The effect of water deficit-stress on plant life is affected by the plant growth period, length and strength of the water

To cite this paper: Abdelmalik AM, TS Alshahrani, AA Al-Qarawi (2021). Enhanced growth and drought resistance in seedlings of Acacia tortilis due to inoculation of arbuscular mycorrhiza fungi and Bacillus subtilis. Intl J Agric Biol 26:750-760 
deficit-stress (Sharma et al. 2020). However, tree seedling stage is the most sensitive phase to the water deficit-stress for many plant species (Arrieta and Suárez 2006), because of their limited root networks that mostly found at the topsoil layer which make them experience further severe water deficiency than large trees and eventually, drought can lead to its destruction completely (Mueller et al. 2005). Seedling's stage of A. tortilis life cycle is the most critical stage; therefore, in this study we investigated the impact of coinoculation of AMF and B. subtilis on growth and tolerance of $A$. tortilis seedlings to water deficit-stress.

\section{Materials and Methods}

\section{Mycorrhizal fungi}

The mycorrhizal fungi in our study consisted of a combination of Funneliformis mosseae (Syn. Glomus mosseae), Rhizophagus intraradices (Syn. Glomus intraradices) and Claroideoglomus etunicatum (Syn. Glomus etunicatum). These AMF fungi were extracted and isolated from the hair roots of Conocarpus erectus trees. AMF species were identified following the protocol defined by Redecker et al. (2013), where spores were separated and observed under computerized compound microscope. The identification process depended on the morphological characteristics of the spores.

\section{Propagation of AMF}

Inoculums of the mycorrhizal fungi were developed for 4 weeks in pots containing Sudan grass (Sorghum sudanense). The source inoculums were taken from the fine roots of Conocarpus erectus trees at the faculty of Agriculture and Food Sciences, King Saud University (KSU) and then placed in autoclaved sandy soils. After that, seeds of the host plant (Sudan grass) were spread in the pots. Pots were irrigated as needed until the host plant (Sudan grass) grown and established and become ready to be applied as inoculums.

\section{Bacterium inoculants preparation}

B. subtilis, was isolated previously from the roots of Acacia seyal Benth trees (Alqarawi et al. 2014; Hashem et al. 2015). The inoculants of $B$. subtilis were prepared in small flasks $(250 \mathrm{~mL})$, each flask has $100 \mathrm{~mL}$ of nutrient medium and then flasks were incubated on a shaker for three days at $25^{\circ} \mathrm{C}$. Afterwards, the bacillus suspensions were adjusted to $3.6 \times$ $109 \mathrm{cfu} \mathrm{mL}^{-1}$.

\section{Plant culture and growth conditions}

Seeds of A. tortilis were provided by Forestry Research Centre, Khartoum, Soba, The Republic of the Sudan. The experiment was carried out in the shade house, Faculty of Food and Agriculture Sciences, KSU, from March to June. Seeds were sown in a plastic pot $(50 \mathrm{~cm}$ height and $16 \mathrm{~cm}$ diameter). Pots were filled by a sandy loamy soil (3:1 v/v), with following characteristics: $0.42 \%$ of organic carbon with $0.075-0.10 \mathrm{~mm}$ particle size. The procedures described by Sommers (1982) and Miller (1987) was used for particle size and organic carbon analysis.

In each pot two seedlings were established in sterilized sandy loam soil. Pots were inoculated with AMF, Bacillus, and co-inoculants (AMF + Bacillus). Inoculation of AMF was done to the soil before seeding process. For bacillus treatment, seeds were dipped in the B. subtilis suspensions for 10 minutes and then talc powder was added as an adhesive material. After that, seeds were removed from suspension and dried at room temperature and then planted in the soil. Further suspension was added to the soil of Bacillus and combination treatments to increase cell number of Bacillus in the seedling's rhizosphere. Another group of seedlings was established under the same environment but without inoculants (control). Seedlings were irrigated frequently until the second true leaf was shown, after that, the seedlings were exposed to four irrigation intervals where pots irrigated by $250 \mathrm{~mL}$ of water every 1, 2, 3 and 4- weeks watering interval (water-deficit treatments).

\section{Design of the experiment and layout}

A split-plot arrangement in randomized complete block design was used to set up the experiment. Treatment consisted of four drought intervals (1, 2, 3 and 4 weeks) and four groups of microorganism's treatments; control (no microorganisms), AMF, B. subtilis and co-inoculants (AMF + Bacillus) with four replications (pot) per treatment.

\section{Root colonization by mycorrhizal fungi}

AMF were extracted from root hairs samples of AMF and coinoculants treatments following the method defined by Daniels and Skipper (1982) and modified by Utobo et al. (2011). The roots were well washed with distilled water to remove the soil particles adherent to it, then washed with $\mathrm{KOH}(10 \%)$ and afterward stained with trypan blue in lactophenol, as followed by Phillips and Hayman (1970). The stained root hairs were cut to small segments, and then checked by a bright microscope at $400 \times 23$ magnification. Mycorrhizal fungi infection (mycelium, vesicles and arbuscules) in root hairs was measured using the following formula:

$$
\% \text { Colonization }=\frac{\text { Total number of AMF positive segments }}{\text { Total number of segments studied }} \times 100
$$

\section{Spore extraction}

AMF Spores were separated using wet sieving and decanting method (Gerdemann and Nicolson 1963). A $100 \mathrm{~g}$ of soil samples were air dried and $800 \mathrm{~mL}$ of water was added to generate soil suspension. The suspension was filtered using gradual sieves. Then, suspension was filtered through gridded Whatman filter paper No. 1. The filter paper was 
tested under microscope at $2.5 \times 10$ magnification and then spores number was recorded.

\section{Measurements of areal and root part traits}

Roots fresh weight, stems, and leaves were separated from each other and were weighed using a digital balance scale. Then, the roots, vegetative part (stem and leaves) were individually dried at $75^{\circ} \mathrm{C}$ for $48 \mathrm{~h}$ to achieve dry weights.

The following traits were measured during experiment: height of plant $(\mathrm{cm})$ from the cotyledon scars to the seedling apex using a ruler, seedling stem diameter $(\mathrm{mm})$ at the cotyledon scar using a digital caliper $( \pm 0.04 \mathrm{~mm})$, leaves number, leaf area by using portable leaf area meter (Model CI202, CID, Bio-Science, Camas, USA) and branches number.

Seedlings were smoothly taken out from the soil, and then roots were separated from the shoot. Seedling roots were washed well from the adhesive soil and then spread gently over a scanner device connected with a computer and then and scanned at 600 dots per inch. The root images were saved in TIFF format to be evaluated and measured by a computer software. The root traits (total root length $(\mathrm{cm})$, root surface area, root volume and root dimeter were measured using WinRhizo Pro software (Regent Instruments Inc and Christian 1996).

\section{Estimation of chlorophyll $a$ and $b$}

Fresh leaf samples were collected from each treatment. Sample of fresh leaves with $0.5 \mathrm{~g}$ per treatment was weighted using digital balance scale and then placed at glass tube. Each tube filled with $5 \mathrm{~mL}$ of diemethyl formamide and left for 24 $\mathrm{h}$ at room temperature. After $24 \mathrm{~h}$, leaf extracts were filtered and placed in spectrophotometer cuvette and read absorption at $664 \mathrm{~nm}$, for chlorophyll a and at $620 \mathrm{~nm}$ for Chlorophyll b (Porra et al. 1989).

\section{Estimation of Proline accumulation}

Sadasivam and Manickam (1996) protocol was followed to measure seedlings proline content. Where, small sample from seedlings leaves $(0.5 \mathrm{~g})$ were clipped in the early morning and grounded in mortar and pestle by adding $10 \mathrm{~mL}$ of $3 \%$ sulphosalicyclic acid and the resulted homogenate was centrifuged at $18000 \mathrm{~g}$ for $1 \mathrm{~h}$ and purified. Then, $2 \mathrm{~mL}$ of filtered solution were added in test containers to glacial acetic acid $(2 \mathrm{~mL})$ and acid ninhydrin $(2 \mathrm{~mL})$ and test containers were watery bathed for $1 \mathrm{~h}$ at $100^{\circ} \mathrm{C}$, followed by ice bath. The reaction blend was vortexed with toluene $(4 \mathrm{~mL})$. Layer of toluene was separated, and the absorbance was measured using spectrophotometer at $520 \mathrm{~nm}$ (Genesis 10-S, Thermo Fisher Scientific, Madison, USA). A standard curve of proline was used to identify proline accumulation.

\section{Statistical analysis of the data}

Analysis of variance (ANOVA) was used to analyze the data and means were separated using Fisher's least significance difference test (LSD) at $P<0.05$. Statistical analyses were done using the SPSS software package version 22.0.

\section{Results}

\section{Infection of $A$. tortilis roots by AMF}

AMF colonization rate: AMF obviously colonized the roots of A. tortilis at mycorrhizal and co-inoculants treatments (Fig. 1). The highest colonization rate was recorded at co-inoculant treatments at all irrigation interval. The greatest colonization percentage $(93.3 \%$ for mycelium, $77.2 \%$ for vesicle, and $68.1 \%$ for arbuscular) which was recorded at co-inoculant treatment at 1-week irrigation intervals. The lowest colonization rate $50 \%$ for mycelium, $14.7 \%$ for vesicle, and $17.9 \%$ for arbuscular) at 4, 2, 3-weeks irrigation interval respectively (Table 1 ).

Spores density: The spores' total densities varied between irrigation intervals and between AMF and co-inoculant treatment. The highest number of spores was (104 spores 10 $\mathrm{g}^{-1}$ ) which recorded at co-inoculant treatment, and at 1-week watering interval, however, the lowest spore's number was (30 spores $10 \mathrm{~g}^{-1}$ ) which found at the AMF treatment at 4weeks irrigation interval (Table 1).

Effect of inoculants on shoot fresh and dry weight of $A$. tortilis seedlings

Results of statistical analysis showed significant impact for inoculants treatments (co-inoculant, AMF, Bacillus) on shoot fresh and dry weight in all irrigation intervals compared to control (Table 2). The seedlings treated with co-inoculants showed the highest averages for shoot fresh and dry weights in all irrigation intervals, followed by AMF-treated seedlings, and then $B$. subtilis treated seedlings. On other hand, control seedlings showed the lowest shoot fresh and dry weights (Table 2). In comparison to the control seedlings, co-inoculant increased shoot fresh weight by $(207.07,495.44,916.77$ and $792.65 \%)$ and shoot dry weight by $(177.43,1482.97,891.17$ and $651.24 \%$ ) at $1,2,3$ and 4weeks irrigation intervals respectively. However, AMF treated seedlings increased by $140.40,1181.20,659.02$ and $626.95 \%$, (for shoot fresh weight) and 148.44, 980.69, 668.38 and $636.93 \%$, (for shoot dry weight), at 1, 2, 3 and 4-weeks irrigation interval. For $B$. subtilis treated seedlings, the percentage of increments were $26.63,736,5.62$ and $5.32 \%$ (for shoot fresh weight) and 1.49, $511.02,2.83$ and $5.68 \%$ (for shoot dry weight), at 1, 2, 3 and 4-weeks irrigation interval.

Effect of inoculants on vegetative growth of $A$. tortilis seedlings

Statistical analysis indicated that co-inoculants treatment significantly affected seedlings height, leaf number and leaf area of the seedlings, compared to the control (Table 3). In the different irrigation interval, seedlings treated with co- 
Enhancing Drought tolerance in Acacia tortilis Seedlings / Intl J Agric Biol, Vol 26, No 6, 2021

Table 1: Percentages of AMF colonization and spore's number in the roots and rhizosphere of A. tortilis seedlings

\begin{tabular}{llllll}
\hline \multirow{2}{*}{ Irrigation interval (weeks) } & Inoculant treatment & \multicolumn{3}{c}{ AMF Colonization rate (\%) } & Spores' numbers \\
\cline { 3 - 5 } & & Mycelium & Vesicle & Arbuscular & \\
\hline 1 & AMF & 76.9 & 27.0 & 47.8 & 76 \\
2 & Co-inoculant & 93.3 & 77.2 & 68.1 & 104 \\
3 & AMF & 66.6 & 20.0 & 26.6 & 52 \\
& Co-inoculant & 74.1 & 14.7 & 55.2 & 60 \\
4 & AMF & 63.3 & 20.0 & 20.0 & 48 \\
& Co-inoculant & 85.5 & 25.8 & 17.9 & 46 \\
& AMF & 50.0 & 23.3 & 30.0 & 30 \\
\end{tabular}

Table 2: Effect of co-inoculant on shoot fresh and dry weights of $A$. tortilis seedlings under different irrigation intervals

\begin{tabular}{llll}
\hline Irrigation interval (weeks) & Inoculant treatment & Shoot fresh weight $(\mathrm{g} /$ plant $)$ (Means \pm SE) & Shoot dry weight $(\mathrm{g} /$ plant $)($ Means \pm SE) \\
\hline 1 & Control & $1.7900 \pm 0.1127 \mathrm{~d}$ & $0.8867 \pm 0.1186 \mathrm{~d}$ \\
& AMF & $4.3033 \pm 0.2603 \mathrm{~b}$ & $2.2033 \pm 0.1090 \mathrm{ab}$ \\
& Bacillus & $2.2667 \pm 0.2624 \mathrm{c}$ & $0.9000 \pm 0.0473 \mathrm{~d}$ \\
& Co-inoculant & $5.4967 \pm 0.1849 \mathrm{ab}$ & $2.4600 \pm 0.1350 \mathrm{a}$ \\
& Control & $0.2500 \pm 0.0416 \mathrm{e}$ & $0.1533 \pm 0.0145 \mathrm{e}$ \\
& AMF & $3.2033 \pm 0.3569 \mathrm{bc}$ & $1.6567 \pm 0.1617 \mathrm{bc}$ \\
& Bacillus & $2.0900 \pm 0.2312 \mathrm{c}$ & $0.9367 \pm 0.1004 \mathrm{~d}$ \\
3 & Co-inoculant & $5.6567 \pm 0.0982 \mathrm{a}$ & $2.4267 \pm 0.1405 \mathrm{a}$ \\
& Control & $0.2367 \pm 0.0133 \mathrm{e}$ & $0.1167 \pm 0.0120 \mathrm{e}$ \\
& AMF & $1.7967 \pm 0.2196 \mathrm{~d}$ & $0.8967 \pm 0.1213 \mathrm{~d}$ \\
& Bacillus & $0.2500 \pm 0.0470 \mathrm{e}$ & $0.1200 \pm 0.0208 \mathrm{e}$ \\
4 & Co-inoculant & $2.4067 \pm 0.3421 \mathrm{c}$ & $1.1567 \pm 0.1172 \mathrm{~cd}$ \\
& Control & $0.2233 \pm 0.0353 \mathrm{e}$ & $0.1167 \pm 0.0203 \mathrm{e}$ \\
& AMF & $1.6233 \pm 0.1849 \mathrm{~d}$ & $0.8600 \pm 0.0924 \mathrm{~d}$ \\
BSD & Bacillus & $0.2633 \pm 0.0524 \mathrm{e}$ & $0.1233 \pm 0.0120 \mathrm{e}$ \\
Sig & Co-inoculant & $1.9933 \pm 0.3023 \mathrm{~d}$ & $0.8767 \pm 0.1091 \mathrm{~d}$ \\
\hline
\end{tabular}

Mean \pm standard errors. Values with same letters differ non-significantly at $(P>0.05)$

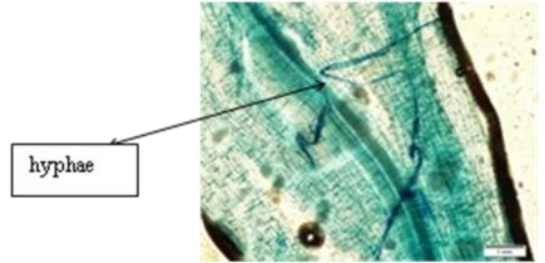

Fig. 1: Infection and colonization of $A$. tortilis roots by AMF

inoculants showed the greatest average of height which was $45.33 \mathrm{~cm}, 49.66 \mathrm{~cm}, 60.00 \mathrm{~cm}$, and $50.66 \mathrm{~cm}$ at $1,2,3$ and $4-$ weeks irrigation interval, respectively. The average of leaf number/plant was also the greatest in the all-different irrigation intervals with average of 34.333 (at 1-week irrigation intervals), 36.33 (at 2-weeks irrigation interval), 41.66 (at 3-weeks irrigation interval), and 17.33 (at 4-weeks irrigation interval). Leaf area also has the highest average in co-inoculants treatment, which was $92.667 \mathrm{~cm}^{2}, 131.85 \mathrm{~cm}^{2}$, $37.663 \mathrm{~cm}^{2}$ and $37.043 \mathrm{~cm}^{2}$, at 1, 2, 3 and 4-week irrigation intervals, respectively (Table 3 ).

\section{Effect of inoculants on root growth of $A$. tortilis seedlings}

Co-inoculants, AMF and Bacillus treatments, significantly improved root fresh and dry weights in A. tortilis seedlings under the different drought conditions. Both co-inoculants and

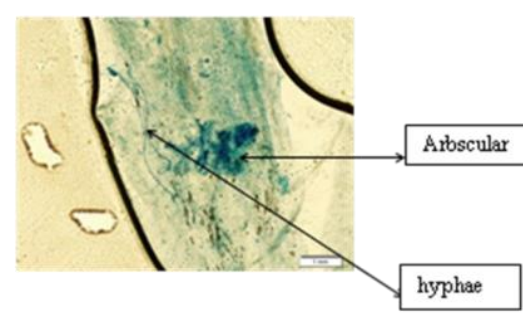

AMF treated seedlings showed the greatest average of shoot fresh and dry weights (Table 4). At 3 and 4-week irrigation intervals, drought decreased root fresh and dry weight of control and bacillus treated seedlings, however co-inoculated and AMF-seedlings were not affected by drought in terms of root fresh and dry weight (Table 4). Similarly, inoculum treatments had positive effect on the root parameters (root length, root surface area, root volume, and root diameter); where AMF and co-inoculants treatments showed the greatest root system at all irrigation intervals (Table 5 and Fig. 2-5).

\section{Effect of inoculants on chlorophyll a and b}

Inoculant's treatments significantly improved the content of chlorophyll-a in the leave of $A$. tortilis seedlings. No significant different was observed in chlorophyll $b$, however, seedlings inoculated with co-inoculants, AMF, and $B$. 
Abdelmalik et al. / Intl J Agric Biol, Vol 26, No 6, 2021

Table 3: Effect of co-inoculant on height, leaf number and leaf area of $A$. tortils seedlings under different irrigation intervals

\begin{tabular}{llllll}
\hline $\begin{array}{l}\text { Irrigation Intervals } \\
\text { (weeks) }\end{array}$ & $\begin{array}{l}\text { Inoculant } \\
\text { treatment }\end{array}$ & $\begin{array}{l}\text { Height }(\mathrm{cm}) \\
(\text { Means } \pm \text { SE) }\end{array}$ & $\begin{array}{l}\text { Leaf number } \\
\text { (Means } \pm \text { SE) }\end{array}$ & $\begin{array}{l}\text { Leaf area }\left(\mathrm{cm}^{2}\right) \\
(\text { Means } \pm \text { SE) }\end{array}$ & $\begin{array}{l}\text { Leaf temperature }{ }^{\circ} \mathrm{C} \\
(\text { Means } \pm \text { SE) }\end{array}$ \\
\hline 1 & Control & $18.667 \pm 4.6667 \mathrm{fg}$ & $13.667 \pm 0.3333 \mathrm{fg}$ & $34.577 \pm 2.1219 \mathrm{ef}$ & $29.933 \pm 0.6936 \mathrm{a}$ \\
& AMF & $54.000 \pm 1.7321 \mathrm{a}$ & $46.000 \pm 1.5275 \mathrm{a}$ & $124.16 \pm 3.0751 \mathrm{a}$ & $26.367 \pm 1.6707 \mathrm{abcd}$ \\
& Bacillus & $29.667 \pm 3.3830 \mathrm{def}$ & $21.667 \pm 1.2019 \mathrm{de}$ & $50.253 \pm 2.1040 \mathrm{~d}$ & $25.067 \pm 0.4910 \mathrm{bcde}$ \\
& Co-inoc & $45.333 \pm 2.1858 \mathrm{abc}$ & $34.333 \pm 1.2019 \mathrm{c}$ & $92.667 \pm 3.1714 \mathrm{~b}$ & $24.033 \pm 1.2811 \mathrm{cde}$ \\
& Control & $21.667 \pm 1.4530 \mathrm{efg}$ & $10.000 \pm 0.5774 \mathrm{gh}$ & $25.827 \pm 1.1526 \mathrm{fg}$ & $29.567 \pm 0.3844 \mathrm{ab}$ \\
& AMF & $39.333 \pm 2.8480 \mathrm{bcd}$ & $27.000 \pm 0.5774 \mathrm{~d}$ & $43.887 \pm 1.2714 \mathrm{de}$ & $28.100 \pm 0.8505 \mathrm{abcd}$ \\
& Bacillus & $32.333 \pm 1.4530 \mathrm{cde}$ & $19.333 \pm 0.6667 \mathrm{ef}$ & $38.133 \pm 0.1764 \mathrm{def}$ & $23.833 \pm 1.0525 \mathrm{de}$ \\
3 & Co-inoc & $49.667 \pm 0.3333 \mathrm{ab}$ & $36.333 \pm 0.3333 \mathrm{bc}$ & $131.85 \pm 3.8031 \mathrm{a}$ & $21.633 \pm 0.5840 \mathrm{e}$ \\
& Control & $12.000 \pm 0.5774 \mathrm{~g}$ & $5.0000 \pm 0.5774 \mathrm{~h}$ & $15.140 \pm 0.1701 \mathrm{gh}$ & $30.667 \pm 0.4256 \mathrm{a}$ \\
& AMF & $43.333 \pm 0.3333 \mathrm{ab}$ & $36.667 \pm 2.0276 \mathrm{bc}$ & $32.703 \pm 2.7629 \mathrm{ef}$ & $29.267 \pm 0.2963 \mathrm{ab}$ \\
& Bacillus & $14.667 \pm 0.8819 \mathrm{~g}$ & $6.0000 \pm 0.5774 \mathrm{~h}$ & $9.1400 \pm 0.3100 \mathrm{~h}$ & $27.700 \pm 0.9539 \mathrm{abcd}$ \\
& Co-inoc & $54.667 \pm 4.6667 \mathrm{a}$ & $41.667 \pm 2.7285 \mathrm{ab}$ & $37.663 \pm 1.6709 \mathrm{def}$ & $26.967 \pm 0.2186 \mathrm{abcd}$ \\
& Control & $13.000 \pm 0.5774 \mathrm{~g}$ & $7.6667 \pm 0.3333 \mathrm{gh}$ & $11.533 \pm 0.7860 \mathrm{~h}$ & $28.533 \pm 1.3836 \mathrm{abcd}$ \\
& AMF & $39.000 \pm 3.0551 \mathrm{bcd}$ & $18.333 \pm 0.8819 \mathrm{ef}$ & $68.973 \pm 4.6321 \mathrm{c}$ & $28.733 \pm 0.2728 \mathrm{abc}$ \\
LSD & Bacillus & $14.333 \pm 0.6667 \mathrm{~g}$ & $10.000 \pm 0.5774 \mathrm{gh}$ & $6.4400 \pm 0.4903 \mathrm{~h}$ & $28.733 \pm 0.5696 \mathrm{abc}$ \\
Sig & Co-inoc & $50.667 \pm 1.8559 \mathrm{ab}$ & $17.333 \pm 0.8819 \mathrm{ef}$ & $37.043 \pm 3.1542 \mathrm{ef}$ & $26.267 \pm 0.2963 \mathrm{abcd}$ \\
\hline
\end{tabular}

Mean \pm standard errors. Values with same letters differ non-significantly at $(P>0.05)$

Table 4: Effect of co-inoculant on root length, root surface area, and root tips number of $A$. tortils seedlings under different irrigation intervals

\begin{tabular}{llll}
\hline Irrigation interval (weeks) & Inoculant treatment & Root fresh weight $(\mathrm{g})$ (Means \pm SE) & Root dry weight $(\mathrm{g})(\mathrm{Means} \pm \mathrm{SE})$ \\
\hline 1 & Control & $2.7167 \pm 0.2554 \mathrm{bc}$ & $1.6933 \pm 0.0736 \mathrm{bcd}$ \\
& AMF & $4.3667 \pm 0.4914 \mathrm{ab}$ & $2.2500 \pm 0.2616 \mathrm{ab}$ \\
& Bacillus & $3.7567 \pm 0.3779 \mathrm{abc}$ & $1.4633 \pm 0.1027 \mathrm{bcd}$ \\
& Co-inoculation & $5.1167 \pm 0.3805 \mathrm{a}$ & $2.2767 \pm 0.1172 \mathrm{ab}$ \\
& Control & $0.1767 \pm 0.0536 \mathrm{~d}$ & $0.1133 \pm 0.0120 \mathrm{f}$ \\
& AMF & $3.6100 \pm 0.3444 \mathrm{abc}$ & $1.7633 \pm 0.1387 \mathrm{bcd}$ \\
& Bacillus & $2.3167 \pm 0.2293 \mathrm{c}$ & $1.0867 \pm 0.0176 \mathrm{cde}$ \\
3 & Co-inoculation & $4.1300 \pm 0.3208 \mathrm{abc}$ & $1.4833 \pm 0.2350 \mathrm{bcd}$ \\
& Control & $0.1533 \pm 0.0120 \mathrm{~d}$ & $0.0800 \pm 0.0115 \mathrm{f}$ \\
& AMF & $5.3167 \pm 0.4667 \mathrm{a}$ & $2.7833 \pm 0.2530 \mathrm{a}$ \\
& Bacillus & $0.2800 \pm 0.0001 \mathrm{~d}$ & $0.1700 \pm 0.0115 \mathrm{ef}$ \\
& Co-inoculation & $3.1533 \pm 0.5128 \mathrm{bc}$ & $1.7767 \pm 0.2826 \mathrm{bcd}$ \\
& Control & $0.2267 \pm 0.0338 \mathrm{~d}$ & $0.1200 \pm 0.0321 \mathrm{f}$ \\
& AMF & $2.3733 \pm 0.3457 \mathrm{c}$ & $1.0033 \pm 0.1586 \mathrm{def}$ \\
BSD & Bacillus & $0.3233 \pm 0.0273 \mathrm{~d}$ & $0.2067 \pm 0.0145 \mathrm{ef}$ \\
Sig & Co-inoculation & $3.9267 \pm 0.6274 \mathrm{abc}$ & $1.9867 \pm 0.2765 \mathrm{abc}$ \\
\hline Mes & & 1.8283 & 0.9614 \\
\hline
\end{tabular}

Mean \pm standard errors. Values with same letters differ non-significantly at $(P>0.05)$

subtilis showed better content of chlorophyll-b than control seedlings (Table 6).

\section{Effect of inoculants on proline accumulation}

The proline accumulation in the leaves of all seedlings improved by increasing the irrigation intervals (Table 7). However, seedlings inoculated with co-inoculants, AMF, and bacillus showed lower content of proline than in control seedlings regardless of the irrigation intervals. This variation was more obvious under varied irrigation intervals.

\section{Discussion}

Responses of the plant to the soil's microbes are the consequence of interaction relationship between plants and microbes found in the soil. It obviously appears from our results that $A$. tortilis seedlings much improved and resisted drought when AMF and their combination with $B$. subtilis were applied.

Inoculation with AMF, Bacillus and co-inoculants improved plant height, leaf number, leaf area, and shoot fresh and dry weights. This improvement could be due to the increased presence of carbohydrates in the shoot part, enhancement of nutrients uptake (Verma et al. 2018) and increase of root system in treated seedlings. Our results in the same line with many studies that found, AMF improves plant growth by increasing nutrients amount in the soil and its absorption to the plant (Naheeda et al. 2020; Ya-Dong et al. 2021).

In general, seedlings inoculated with AMF, bacillus, and co-inoculants showed higher vegetative growth in comparison to the control seedlings. This finding is similar 
Enhancing Drought tolerance in Acacia tortilis Seedlings / Intl J Agric Biol, Vol 26, No 6, 2021

Table 5: Effect of co-inoculant on root length, root surface area, root volume and root diameter of $A$. tortils seedlings under different irrigation intervals

\begin{tabular}{|c|c|c|c|c|c|}
\hline $\begin{array}{l}\text { Irrigation } \\
\text { (Weeks) }\end{array}$ & $\begin{array}{r}\text { interval Inoculant } \\
\text { treatment }\end{array}$ & $\begin{array}{l}\text { Root length }(\mathrm{cm}) \text { (Means } \pm \\
\text { SE) }\end{array}$ & $\begin{array}{l}\text { Root surface area }\left(\mathrm{cm}^{2}\right) \text { (Means } \\
\pm \mathrm{SE})\end{array}$ & $\begin{array}{l}\text { Root volume (Means } \pm \\
\text { SE) }\end{array}$ & $\begin{array}{l}\text { Root diameter (Means } \pm \\
\text { SE) }\end{array}$ \\
\hline \multirow[t]{4}{*}{1} & Control & $414.47 \pm 24.056 b$ & $90.870 \pm 6.9272 b c$ & $1.8367 \pm 0.1135 \mathrm{ce}$ & $0.7233 \pm 0.0318 b c$ \\
\hline & AMF & $697.14 \pm 4.9770 \mathrm{a}$ & $232.31 \pm 34.631 \mathrm{a}$ & $3.5067 \pm 0.2373 \mathrm{ab}$ & $1.1600 \pm 0.0600 \mathrm{a}$ \\
\hline & Bacillus & $554.28 \pm 66.016 \mathrm{ab}$ & $168.39 \pm 13.964 \mathrm{ab}$ & $3.1333 \pm 0.1648 \mathrm{ab}$ & $0.7867 \pm 0.0273 b c$ \\
\hline & Co-inoculation & $618.13 \pm 76.139 a b$ & $160.26 \pm 8.8719 \mathrm{ab}$ & $3.6233 \pm 0.5732 \mathrm{ab}$ & $0.7833 \pm 0.0521 b c$ \\
\hline \multirow[t]{4}{*}{2} & Control & $152.39 \pm 8.5729 c$ & $34.137 \pm 4.4081 b c$ & $0.6933 \pm 0.0751 \mathrm{e}$ & $0.7400 \pm 0.0404 b c$ \\
\hline & AMF & $424.22 \pm 31.882 b$ & $197.06 \pm 60.199 \mathrm{ab}$ & $3.2267 \pm 0.3548 \mathrm{ab}$ & $0.7000 \pm 0.0404 b c$ \\
\hline & Bacillus & $523.64 \pm 83.834 \mathrm{ab}$ & $132.62 \pm 18.121 \mathrm{abc}$ & $1.7533 \pm 0.0120 \mathrm{ce}$ & $0.7500 \pm 0.0643 b c$ \\
\hline & Co-inoculation & $348.25 \pm 15.681 b c$ & $140.03 \pm 9.3029 \mathrm{ab}$ & $4.5767 \pm 0.4421 \mathrm{a}$ & $1.1233 \pm 0.0426 \mathrm{a}$ \\
\hline \multirow[t]{4}{*}{3} & Control & $96.890 \pm 6.5094 c$ & $21.010 \pm 1.1252 \mathrm{c}$ & $0.4167 \pm 0.041 \mathrm{e}$ & $0.6400 \pm 0.0764 b c$ \\
\hline & $\mathrm{AMF}$ & $373.33 \pm 25.584 b c$ & $135.50 \pm 3.0394 \mathrm{abc}$ & $4.3667 \pm 0.1913 a$ & $1.1633 \pm 0.0940 \mathrm{a}$ \\
\hline & Bacillus & $135.13 \pm 12.871 \mathrm{c}$ & $39.097 \pm 3.3340 \mathrm{bc}$ & $0.9033 \pm 0.0736 c e$ & $0.9233 \pm 0.0233 \mathrm{ab}$ \\
\hline & Co-inoculation & $418.36 \pm 33.081 b$ & $160.22 \pm 18.291 \mathrm{ab}$ & $3.4700 \pm 0.7410 \mathrm{ab}$ & $1.1433 \pm 0.0581 \mathrm{a}$ \\
\hline \multirow[t]{4}{*}{4} & Control & $152.38 \pm 6.5865 c$ & $55.617 \pm 9.9760 \mathrm{bc}$ & $1.0233 \pm 0.0433 \mathrm{ce}$ & $0.5867 \pm 0.0176 c$ \\
\hline & AMF & $597.11 \pm 43.652 \mathrm{ab}$ & $146.42 \pm 14.684 a b$ & $2.4000 \pm 0.0651 b c$ & $0.6267 \pm 0.0463 c$ \\
\hline & Bacillus & $152.94 \pm 22.496 c$ & $31.597 \pm 3.8153 b c$ & $0.5200 \pm 0.0557 \mathrm{e}$ & $0.6567 \pm 0.0296 b c$ \\
\hline & Co-inoculation & $567.35 \pm 57.092 \mathrm{ab}$ & $174.36 \pm 7.8967 \mathrm{ab}$ & $4.0100 \pm 0.2201 a$ & $0.7700 \pm 0.0100 b c$ \\
\hline Sig & & $* * * *$ & $* *$ & $* * *$ & $* * *$ \\
\hline
\end{tabular}

Mean \pm standard errors. Values with same letters differ non-significantly at $(P>0.05)$

Table 6: Effect of co-inoculant on chlorophyll-a, chlorophyll-b, and proline accumulation of Acacia tortilis seedlings under different irrigation intervals

\begin{tabular}{|c|c|c|c|}
\hline Irrigation interval (Weeks) & Inoculant treatment & Chlorophyll-a (Means \pm SE) & Chlorophyll-b (Means \pm SE) \\
\hline \multirow[t]{3}{*}{1} & Control & $1.9857 \pm 0.0110 \mathrm{bc}$ & $1.3440 \pm 0.0832 \mathrm{ab}$ \\
\hline & AMF & $2.9003 \pm 0.0454 a$ & $1.7140 \pm 0.0188 \mathrm{ab}$ \\
\hline & Bacillus & $2.9523 \pm 0.0288 \mathrm{a}$ & $1.6060 \pm 0.2441 \mathrm{ab}$ \\
\hline \multirow[t]{4}{*}{2} & Control & $2.2950 \pm 0.0277 b$ & $1.2723 \pm 0.0598 b$ \\
\hline & AMF & $2.3320 \pm 0.0519 b$ & $1.4120 \pm 0.1868 \mathrm{ab}$ \\
\hline & Bacillus & $2.7450 \pm 0.0279 \mathrm{ab}$ & $1.1680 \pm 0.2480 \mathrm{~b}$ \\
\hline & Co-inoculation & $2.6863 \pm 0.2295 \mathrm{ab}$ & $1.5350 \pm 0.0594 \mathrm{ab}$ \\
\hline \multirow[t]{4}{*}{3} & Control & $1.5680 \pm 0.0150 c$ & $1.2320 \pm 0.0576 b$ \\
\hline & $\mathrm{AMF}$ & $1.9073 \pm 0.0135 b c$ & $1.6567 \pm 0.0640 \mathrm{ab}$ \\
\hline & Bacillus & $1.8723 \pm 0.0306 \mathrm{bc}$ & $1.1723 \pm 0.0351 b$ \\
\hline & Co-inoculation & $2.4057 \pm 0.0872 \mathrm{ab}$ & $1.5570 \pm 0.0188 \mathrm{ab}$ \\
\hline \multirow[t]{4}{*}{4} & Control & $1.4153 \pm 0.2040 \mathrm{c}$ & $1.2600 \pm 0.1123 b$ \\
\hline & AMF & $1.5793 \pm 0.0946 \mathrm{c}$ & $1.4537 \pm 0.2041 \mathrm{ab}$ \\
\hline & Bacillus & $1.5987 \pm 0.0345 \mathrm{c}$ & $1.2657 \pm 0.0198 b$ \\
\hline & Co-inoculation & $2.4500 \pm 0.2306 \mathrm{ab}$ & $2.0600 \pm 0.0259 a$ \\
\hline Sig & & $* * *$ & NS \\
\hline
\end{tabular}

Mean \pm standard errors. Values with same letters differ non-significantly at $(P>0.05)$ : NS: not significant

Table 7: Effect of co-inoculant on proline accumulation of Acacia tortilis seedlings under different irrigation intervals

\begin{tabular}{|c|c|c|}
\hline Irrigation interval (Weeks) & Inoculant treatment & Proline content (Means \pm SE) \\
\hline \multirow[t]{4}{*}{1} & Control & $1.1013 \pm 0.1366 \mathrm{abc}$ \\
\hline & AMF & $0.7383 \pm 0.0184 b c$ \\
\hline & Bacillus & $0.5027 \pm 0.0256 c$ \\
\hline & Co-inoculation & $0.5490 \pm 0.0142 \mathrm{c}$ \\
\hline \multirow[t]{4}{*}{2} & Control & $1.0380 \pm 0.1217 \mathrm{abc}$ \\
\hline & AMF & $0.8843 \pm 0.0616 b c$ \\
\hline & Bacillus & $0.7523 \pm 0.1289 b c$ \\
\hline & Co-inoculation & $0.4223 \pm 0.0225 c$ \\
\hline \multirow[t]{4}{*}{3} & Control & $1.3727 \pm 0.0353 \mathrm{ab}$ \\
\hline & $\mathrm{AMF}$ & $0.8570 \pm 0.0749 b c$ \\
\hline & Bacillus & $1.2637 \pm 0.3669 \mathrm{abc}$ \\
\hline & Co-inoculation & $0.9873 \pm 0.0552 \mathrm{abc}$ \\
\hline \multirow[t]{4}{*}{4} & Control & $1.5873 \pm 0.2492 \mathrm{a}$ \\
\hline & $\mathrm{AMF}$ & $0.8850 \pm 0.0111 b c$ \\
\hline & Bacillus & $0.8840 \pm 0.0341 b c$ \\
\hline & Co-inoculation & $0.7677 \pm 0.0528 b c$ \\
\hline LSD $_{0.05}$ & & 0.7255 \\
\hline
\end{tabular}

Mean \pm standard errors. Values with same letters differ non-significantly at $(P>0.05)$ : NS: not significant 


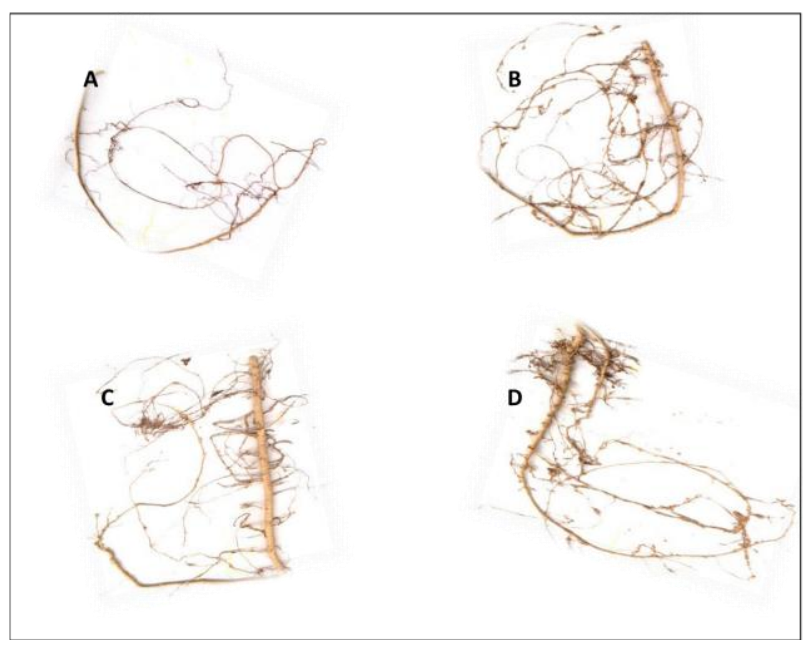

Fig. 2: Effect of inoculants on root architecture of Acacia tortilis seedlings at 1-week irrigation intervals

A: Control treatment; B: Bacillus subtilis treatment; C: AMF treatment; D: coinoculation treatment

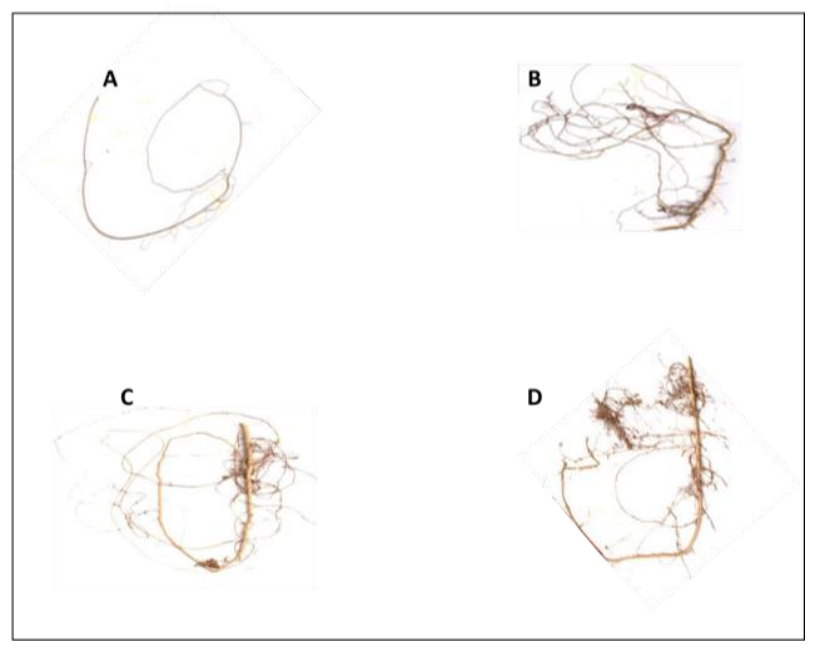

Fig. 3: Effect of inoculants on root architecture of Acacia tortilis seedlings at 2-weeks irrigation intervals

A: Control treatment; B: Bacillus subtilis treatment; C: AMF treatment; D: coinoculation treatment

with our previous results in other acacia species (Abdelmalik et al. 2020). Addition of B. subtilis strain (pf4) (Anand et al. 2010), to plant resulted in a significant vigor index, shoot height and root length (Gowtham et al. 2020). Bacillus species can form endospores that are extremely resilient to harsh environmental conditions and can also produce metabolites that increase growth and vigor of plant. Also, many exopolysaccharides can be produced by bacillus which help water uptake by plant roots (Hashem et al. 2019). Zaidi et al. (2009) stated that B. subtilis acts directly involved in the dissolution of phosphorous and plays a synergistic role with the AMF. AMF alone increased the growth rate by $49.4 \%$; however, when combined with $B$. subtilis, growth rate

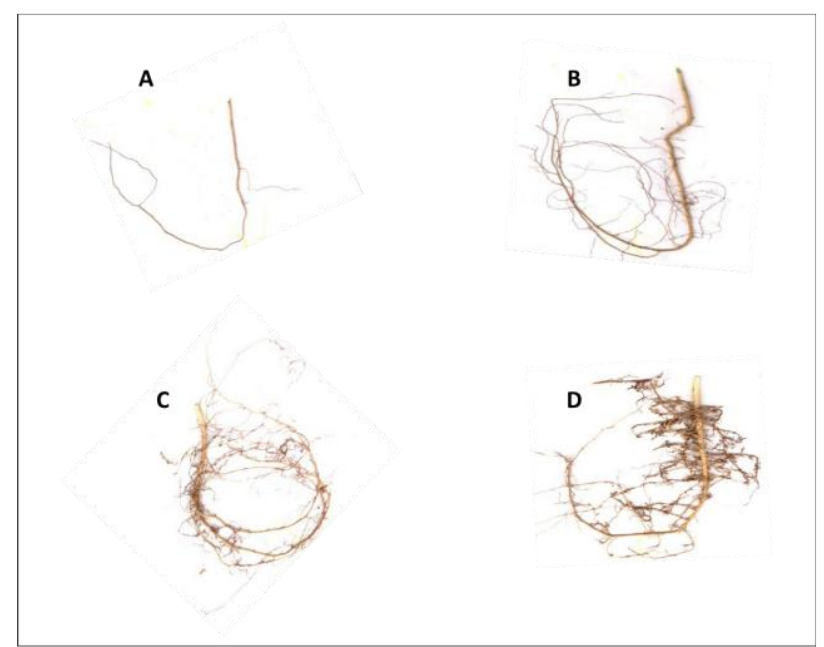

Fig. 4: Effect of inoculants on root architecture of Acacia tortilis seedlings at 3-weeks irrigation intervals

A: Control treatment; B: Bacillus subtilis treatment; C: AMF treatment; D: coinoculation treatment

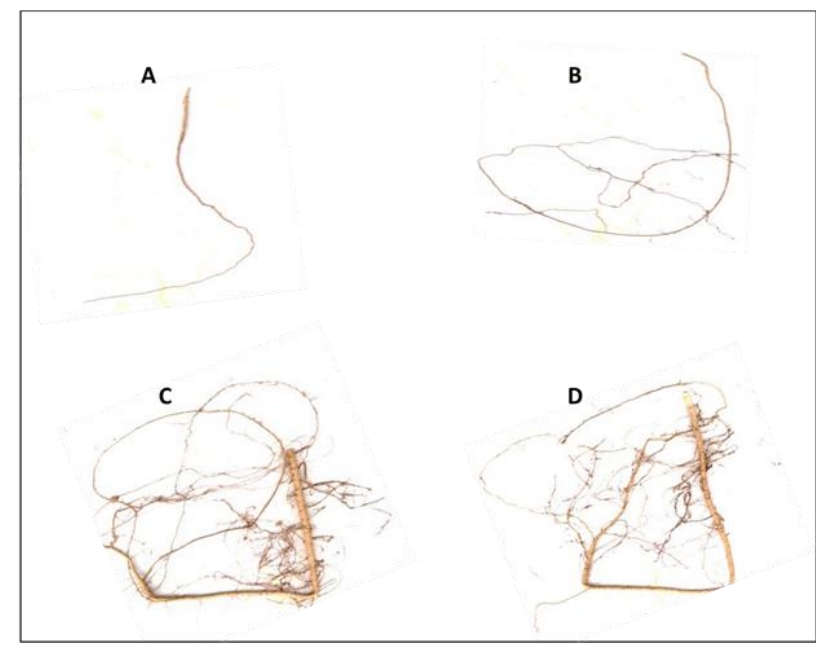

Fig. 5: Effect of inoculants on root architecture of Acacia tortilis seedlings at 4-weeks irrigation intervals

A: Control treatment; B: Bacillus subtilis treatment; C: AMF treatment; D: coinoculation treatment

increased by $59.5 \%$ (Alam et al. 2011). The combined application of AMF and B. subtilis has a synergistic role and leads to promotion of plant growth (Hashem et al. 2019).

Results indicated to varied positive effect for AMF, coinoculant and $B$. subtilis, in the different irrigation intervals and even at severe water-deficit conditions the inoculants showed positive effect on seedlings growth. Microorganisms have tremendous capabilities to reduce environmental stress and their interactions with plants, so that they offer both a local and systemic defense under various environmental stresses (Chialva and Bonfante 2018; Khoshru et al. 2020). Mycorrhizal fungi regulate and improve plant growth when exposed to harsh environmental conditions, where they 
significantly enhanced the growth (Yadav et al. 2018; Xiao et al. 2019; Abdelmalik et al. 2020; Yasser et al. 2021) and biomass of tobacco plants under normal conditions and mitigated the decline caused by water deficit-stress (Begum et al. 2020). In this regard, that inoculation of Onobrychisvicii folias seedlings with AMF reduces the damage resulted from water deficit-stress and improved the water deficit-stress resistance up to forty days (Kong et al. 2014). Interactions between AMF and plant growth promoting rhizobacteria (PGPR) in the plant rhizosphere has a synergistic role which improve growth and quality of the plant (Khalid et al. 2017). PGPR can greatly enhance plant growth and show beneficial interaction between plant and microbes. B. subtilis enhances stress tolerance in plants by stimulating the expression of stress response genes, hormones and metabolites related with drought stress (Lee et al. 2014; Hashem et al. 2019).

AMF, co-inoculants, and $B$. subtilis treated seedlings showed greater root length, root surface area, root diameter, and root volume than non-treated seedlings at all irrigation intervals, which would enable inoculated plants to explore great volume of rhizosphere and hence more nutrients availability to the seedlings. It clear that, the effects of AMF, bacillus, and co-inoculants on root morphology might be an important reason for enhanced nutrients uptake for the treated seedlings. Our results agree with a number of studies which reported that mycorrhizal fungi alters root morphology and increases plant tolerance to the severe environmental conditions (Khanna et al. 2019). Largest root morphology result in better nutrient uptake (Ya-Dong et al. 2021) and enhanced water relationships in the plants (Pallavi and Sharma 2021). The mixed inoculants (B. subtilis + AMF) improved root biomass and plant survival rate in comparison to those caused by sole inoculations and noninoculated plants (Ibrahim et al. 2019). The combined use of PGPR may have a synergic effect on decreasing contrasting stress factors. The application of PGPR with useful fungi in farming is a suitable use in some stressful conditions (Deepmala et al. 2019; Hassan and Bernard 2020). Occurrence of PGPR is highly linked with plant rhizosphere and positive direct and indirect impacts on plant development; like a decline in environmental stress is reported. Bacillus species can make endospores that are tremendously resilient to severe environmental conditions and also can produce metabolites that motivate plant development and fitness (Hashem et al. 2019).

Inoculum's treatments were found to have significant contribution in the improvement of chlorophyll-a and $\mathrm{b}$ in $A$. tortilis seedlings under different irrigation intervals. The improvement of chlorophyll content in AMF and coinoculated seedlings can be justified by the availability of nutrients and metabolism in the plant. However, AMF improve nutritional status of plants by absorbing and translocating mineral nutrients beyond rhizospheric zone (Rouphael et al. 2015). Microorganisms were found to improve the content of plant chlorophyll under normal and water deficit-stress conditions. Co-inoculated plants have higher chlorophyll content compared to single inoculants of AMF or bacteria (Mehdi et al. 2018). Also, the findings reported by Kim et al. (2010) and Berta et al. (2014) proved that co-inoculation (AMF and bacteria) increased the levels of chlorophyll content in plants leaves. The positive effect of AMF was extensively reported by scientists. Various research results explained that the association of microorganisms to the plant can change its physiological growth under many stress conditions (Xiaoying et al. 2014). Mycorrhizal inoculation highly improved the content of chlorophyll-a, b, and total chlorophyll (Naheeda et al. 2020) in the Erythrina variegata leaves. Yooyongwech et al. (2013) and Fang et al. (2018), showed that AMF symbiosis under water deficit conditions enhances chlorophyll fluorescence in woody tree nut species. Sonal et al. (2018) found that total chlorophyll content was more in AMF maize seedlings when compared to non-treated plants where AMF- maize seedlings had double of chlorophyll content as compared to control maize seedlings. AMF colonization could promote the synthesis of chlorophyll and carotenoid thereby enhancing the photosynthesis and biomass accumulation in plants through increasing the root absorption area and root activity, support the absorption and transport of water and other nutrients or mineral elements such as $\mathrm{P}, \mathrm{K}, \mathrm{Mg}$, and $\mathrm{Mn}$ (Baslam et al. 2013). Also, in mycorrhizal plants the increase of chlorophyll contents can be associated with increased $\mathrm{P}$ and $\mathrm{Mg}$ uptake (Zhu et al. 2014).

Proline concentration increased greatly in the leaves of water deficit-stressed and non-inoculated seedlings compared to the well-watered and inoculated seedlings. The lower proline content in AMF and co-inoculation seedlings is an indicator of good drought tolerance of plant (RuizLozano 2003), therefore, the low content of proline in the inoculated seedlings in this study was linked with good seedlings drought tolerance that is induced by AMF and coinoculant treatments. This finding is in agreement with Yooyongwech et al. (2013), where they stated that, AMF and co-inoculation in different plant species reduces proline content when water level is limited. The work done by Doubkova et al. (2013), explained that, when the concentration of proline increases in response to drought stress, a lower proline accumulation has been observed in AMF- plants. In the same way, Wu and Xia (2006) reported that, the content of proline was reduced significantly in orange seedlings inoculated by AMF under water stress conditions. The study conducted by Hazzoumi et al. (2015) reported that leaf proline accumulation was greater in nonAMF plants than AMF-plants under water deficit-stress. The synergistic effects between the bacillus and AMF were reported to increase nutritional status of inoculated plants and thus stimulate the plants resistance to the water deficitstress (Ibrahim et al. 2019). The changes made by PGPR on root elasticity are one of the essential steps to enhanced tolerance to water shortage (Dimkpa et al. 2009). PGPR enhances the plant cell membranes by stimulating the 
antioxidant system and increasing drought tolerance of many plant species (Gusain et al. 2015). Furthermore, AMF plants mostly had better leaf water status and high root volume, thus, plants suffer less water deficit and consequently had lower proline accumulation. The lower proline accumulation in the AMF plants may derive from the integration of the inhibition of glutamate synthetic pathway of proline with an enhancement of proline degradation (Zou et al. 2013).

\section{Conclusion}

Several soil microorganisms positively affect the growth and drought tolerance of $A$. tortilis seedlings, especially in the early stages of their growth, even when exposed to severe water deficit-stresses. The addition of inoculants in general and co-inoculants and AMF in particular resulted in increases in vegetative growth rates (fresh and dry weight, height, number of leaves, leaf area) and root traits (fresh and dry weight, root length, root surface area, root volume). In addition, the inoculants led to an improvement in some physiological characteristics such as chlorophyll-a and b. Also, inoculums reduced proline concentration levels in water deficit-exposed seedlings, and therefore improved seedlings drought tolerance and reduced damage resulting from water deficit due to more water content in inoculated plants. It can be said that, changes in proline levels are a response to tolerance or avoidance for water deficit. Based on the attained results, the inoculants can be used for $A$. tortilis seedlings, especially during the establishment stage. This will assist in the success of afforestation programs in the dry areas.

\section{Acknowledgments}

The authors thank the Deanship of Scientific Research, King Saud University, Saudi Arabia for supporting this work.

\section{Author Contributions}

AM, T S, and AA planed the experiment. AM conducted the experiment, data measurements and analysis, and wrote the first draft. TS analyzed root data and supervised all work. AA supervised the work.

\section{Conflicts of Interest}

All authors declare no conflicts of interest

\section{Data Availability}

Data presented in this study will be available on a fair request to the corresponding author

\section{Ethics Approval}

Not applicable in this paper

\section{References}

Abdelmalik AM, TS Alsharani, AA Al-Qarawi, AI Ahmed, IM Aref (2020). Response of growth and drought tolerance of Acacia seyal Del. seedlings to arbuscular mycorrhizal fungi. Plant Soil Environ 66:264 271

Alam M, A Khaliq, A Sattar, RS Shukla, M Anwar, S Dharni (2011). Synergistic effect of arbuscular mycorrhizal fungi and Bacillus subtilis on the biomass and essential oil yield of rose-scented geranium (Pelargonium graveolens). Soil Sci 57:889-898

Alqarawi AA, EFA Allah, A Hashem (2014). Alleviation of salt-induced adverse impact via mycorrhizal fungi in Ephedra aphylla Forssk. $J$ Plant Interact 9:802-810

Amanda MS, JS Jennifer, BJ Robert (2016). Quantifying drought-induced tree mortality in the open canopy wood lands of central Texas. Rem Sens Environ 181:54-64

Anand M, M Naik, G Ramegowda, G Rani (2010). Biocontrol and plant growth promotion activity of indigenous isolates of Pseudomonas fluorescens. J Mycopathol Res 48:45-50

Andersen GL (2012). Vegetation and management regime continuity in the cultural landscape of the eastern desert, pp:126-139. In: The History of the Peoples of the Eastern Desert Cotsen Institute of Archaeology. Barnard H, K Duistermaat (Eds). Monograph 73. Cotsen Institute of Archaeology, University of California, Los Angeles, California, USA

Arrieta S, F Suarez (2006). Marginal holly (Lexa quifolium) populations in Mediterranean central Spain are constrained by a low-seedling recruitment. Flor Morphol Distrib Funct Ecol Plants 201:152-160

Baslam M, R Esteban, JI Garcia-Plazaola, N Goicoechea (2013). Effectiveness of arbuscular mycorrhizal fungi (AMF) for inducing the accumulation of major carotenoids, chlorophylls and tocopherol in green and red leaf lettuces. Appl Microbiol Biotechnol 97:3119-3128

Begum N, MA Ahangeer, L Zhang (2020). AMF inoculation and phosphorus supplementation alleviates drought induced growth and photosynthetic decline in Nicotiana tabacum by up-regulating antioxidant metabolism and osmolyte accumulation. Environ Exp Bot 176:104088

Berta G, A Fusconi, A Trotta (2014). A mycorrhizal infection and the morphology and function of root systems. Environ Exp Bot 33:159-173

Bhuyan U, C Zang, A Menzel (2017). Different responses of multispecies tree ring growth to various drought indices across Europe. Dendrochronologia 44:1-8

Chialva M, P Bonfante (2018). Plant microbiota: from model plants to Mediterranean crops. Phytopathol Mediterr 57:123-145

Daniels BA, HD Skipper (1982). Methods for the recovery and quantitative estimation of propagules from soil, pp:29-35. In: Methods and Principles of Mycorrhizal Research. Schenck NC (Ed). American Phytopathological Society, St. Paul, Minnesota, USA

Deepmala P, L Rafiq, K Salim, KK Koul (2019). Isolation, screening and molecular characterization of free-living bacteria of potato (Solanum tuberosum $\mathrm{L}$.) and their interplay impact on growth and production of potato plant under mycorrhizal association. Sci Hortic 252:388-397

Díaz-Zorita M, MV Fernández-Canigia (2009). Field performance of a liquid formulation of Azospirillum brasilense on dryland wheat productivity. Eur J Soil Biol 45:3-11

Dimkpa C, T Weinand, F Asch (2009). Plant-rhizobacteria interactions alleviate abiotic stress conditions. Plant Cell Environ 32:1682-1694

Doubkova P, E Vlasakova, R Sudova (2013). Arbuscular mycorrhizal symbiosis alleviates drought stress imposed on Knautia arvensis plants in serpentine soil. Plant Soil 370:149-161

Fang S, Y Tao, Y Zhang, F Kong, Y Wang (2018). Effects of metalaxyl enantiomers stress on root activity and leaf antioxidant enzyme activities in tobacco seedlings. Chirality 30:469-474

Gerdemann J, TH Nicolson (1963). Spores of mycorrhizal Endogone species sex tracted from soil by wet sieving and decanting. Trans Brit Mycol Soc 46:235-244

Gowtham HG, SS Brijesh, M Murali, N Shilpa, P Melvin, KN Mohammed Aiyaz, Amruthesh, SR Niranjana (2020). Induction of drought tolerance in tomato upon the application of ACC deaminase producing plant growth promoting rhizobacterium Bacillus subtilis Rhizo SF 48. Microbiol Res 234:126422 
Gusain YS, US Singh, AK Sharma (2015). Bacterial mediated amelioration of drought stress in drought tolerant and susceptible cultivars of rice (Oryza sativa L.). Afr J Biotechnol 14:764-773

Hashem A, B Tabassum, EF Abd-Allah (2019). Bacillus subtilis: A plantgrowth promoting rhizobacterium that also impacts biotic. Saud J Biol Sci 26:1291-1297

Hashem A, EFA Allah, AA Alqarawi, A Al-huqail, A Asma, SR Alshalawi, S Wirth, D Egamberdieva (2015). Impact of plant growth promoting Bacillus subtilis on growth and physiological parameters of Bassia indica grown under salt stress. Pak J Bot 47:1735-1741

Hassan E, RG Bernard (2020). Halotolerant plant growth-promoting bacteria: Prospects for alleviating salinity stress in plants. Environ Exp Bot 178:104-124

Hazzoumi Z, Y Moustakime, E Elharchli, KA Joutei (2015). Effect of arbuscular mycorrhizal fungi (AMF) and water stress on growth, phenolic compounds, glandular hairs, and yield of essential oil in basil (Ocimum gratissimum L). Chem Biol Technol Agric 2; Article 10

Ibrahim MK, E Eid, HH Mohamed, AA Abbas, AA Salem, A Nevin, A Maha, MS Ghulam, F Chen (2019). Use of plant growth promoting rhizobacteria (PGPR) and mycorrhizae to improve the growth and nutrient utilization of common bean in a soil infected with white rot fungi. Ecotoxicol Environ Saf 171:539-548

Javaid A (2009). Arbuscular mycorrhizal mediated nutrition in plants. J Plant Nutr 32:1595-1618

Khalid M, D Hassani, M Bilal, F Asad, D Huang (2017). Influence of biofertilizer containing beneficial fungi and rhizospheric bacteria on health promoting compounds and antioxidant activity of Spinacia oleracea L. Bot Stud 58:35-44

Khanna K, SK Kohli, P Ohri, R Bhardwaj, AA Al-Huqail, MH Siddiqui, GS Alosaimi, P Ahmad (2019). Microbial fortification improved photosynthetic efficiency and secondary metabolism in Lycopersicon esculentum plants under cd stress. Biomolecules 9:1-20

Khoshru B, S Moharramnejad, NH Gharajeh, BA Lajayer, M Ghorbanpour (2020). Plant microbiome and its important in stressful agriculture. In: Plant Microbiome Paradigm. Springer Nature Switzerland AG

Kim K, W Yim, P Trivedi, M Madhaiyan, HPD Boruah, MR Islam, G Lee, T Sa (2010). Synergistic effects of inoculating arbuscular mycorrhizal fungi and Methylobacterium oryzae strains on growth and nutrient uptake of red pepper. Plant Soil 327:429-440

Kondoh S, H Yahata, T Nakashizuka, M Kondh (2006). Site-specific variation in vessel size, growth drought tolerance of broad-leaved tress in semi-arid regions of Kenya. Tree Physiol 26:899-904

Kong J, Z Pei, M Du, G Sun, X Zhang (2014). Effects of arbuscular mycorrhizal fungi on the drought resistance of the mining area repair plant Sainfoin. Intl J Min Sci Technol 24:485-489

Lee SW, SH Lee, K Balaraju, KS Park, KW Nam, JW Park, K Park (2014). Growth promotion and induced disease suppression of four vegetable crops by a selected plant growth-promoting rhizobacteria (PGPR) strain Bacillus subtilis 21-1 under two different soil conditions. Acta Physiol Plantarum 36:1353-1362

Liddycoat SM, BM Greenberg, DJ Wolyn (2009). The effect of plant growthpromoting rhizobacteria on asparagus seedlings and germinating seeds subjected to water stress under greenhouse conditions. Can J Microbiol 55:388-394

Maarten DB, G Donald, O Mohamed, C Wim (2015). Influence of scattered Acacia trees on soil nutrient levels in arid Tunisia. J Arid Environ 122:161-168

Mehdi G, E Hassan, AA Hossein (2018). Improvement of growth and yield of maize under water stress by co-inoculating an arbuscular mycorrhizal fungus and a plant growth promoting rhizobacterium together with phosphate fertilizers. J Agric Ecosyst Environ 258:59-70

Miller WP (1987). A micro-pipette method for soil mechanical analysis. Commun Soil Sci Plant Anal 18:1-15

Mueller RC, CM Scudder, ME Porter, RT Trotter, CA Gehring, TG Whitham (2005). Differential tree mortality in response to severe drought: evidence for long-term vegetation shifts. $J$ Ecol 93:1085-1093

Nadeem SM, M Ahmad, ZA Zahir, A Javaid, M Ashraf (2014). The role of mycorrhizae and plant growth promoting rhizobacteria (PGPR) in improving crop productivity under stressful environments. Biotechnol Adv 32:429-448
NaheedaB, AAMuhammad,ZLixin (2020). AMF inoculation and phosphorus supplementation alleviates drought induced growth and photosynthetic decline in Nicotiana tabacum by up-regulating antioxidant metabolism and osmolyte accumulation. Environ Exp Bot 176; Article 104088

Pallavi, AK Sharma (2021). Role of arbuscular mycorrhizal fungi in amelioration of drought stress in crop plants. In: Microbes and Signaling Biomolecules Against Plant Stress. Sharma A (Ed) Rhizosphere Biology. Springer, Singapore

Phillips JM, DS Hayman (1970). Improved procedures for clearing roots and staining parasitic and vesicular-arbuscular mycorrhizal fungi for rapid assessment of infection. Tran Brit Mycol Soc 55:158-161

Porra RJ, WA Thompson, PE Kriedemann (1989). Determination of accurate extinction coefficients and simultaneous equations for assaying chlorophylls-a and $b$ extracted with four different solvents: verification of the concentration of chlorophyll standards by atomic absorption spectrometry. Biochem Biophys Acta 975:384-394

Rapparini F, J Penuelas (2014). Mycorrhizal fungi to alleviate drought stress on plant growth. In: Use of Microbes for the Alleviation of Soil Stresses, pp: 21-42. Miransari M (Ed). Springer, New York, USA

Regent Instruments Inc, M Christian (1996). MacRHIZO and WinRHIZO Image Analysis System for Root Measurement. Quebec, Canada

Redecker D, A Schüßler, H Stockinger, SL Stürmer, JB Morton, C Walker (2013). An evidence-based consensus for the classification of carbuncular mycorrhizal fungi (Glomeromycota). Mycorrhiza 23:515531

Richard CT (2016). The impacts of droughts in tropical forests. Trends Plant Sci 21:584-593

Rouphael Y, P Franken, C Schneider, D Schwarz, M Giovannetti, M Agnolucci (2015). Arbuscular mycorrhizal fungi act as biostimulants in horticultural crops. Sci Hortic 196:91-108

Ruiz-Lozano JM (2003). Arbuscular mucorrhizal symbiosis and alleviation of osmotic stress: new perspectives for molecular studies. Mycorrhiza 13:309-317

Sadasivam S, A Manickam (1996). Biochemical Methods, $2^{\text {nd }}$ Edition, pp 107-109. New Age International Publishers Ltd, New Delhi, India

Sharf W, A Javaid, A Shoaib, IH Khan (2021). Induction of resistance in chili against Sclerotium rolfsii by plant growth promoting rhizobacteria and Anagallis arvensis. Egypt J Biol Pest Cont 31:1-11

Sharma A, J Wang, D Xu, S Tao, S Chong, D Yan, Z Li, H Yuan, B Zheng (2020). Melatonin regulates the functional components of photosynthesis, antioxidant system, gene expression, and metabolic pathways to induce drought resistance in grafted Carya cathayensis plants. Sci Total Environ 713:1-13

Sommers (1982). Total carbon, organic carbon, and organic matter. In: Chemical and Microbiological Properties. Methods of Soil Analysis Part 2, $2^{\text {nd }}$ Edition, pp: 539-580. Madison, American Society of Agronomy and Soil Science Society of America

Sonal M, P Mahaveer, J Anjana (2018). Improved photosynthetic efficacy of maize (Zea mays) plants with arbuscular mycorrhizal fungi (AMF) under high temperature stress. J Photochem Photobiol B: Biol 180:149-154

Utobo EB, EN Ogbodo, AC Nwogbaga (2011). Techniques for extraction and quantification of arbuscular mycorrhizal fungi. Libyan Agric Res Center J 2:68-78

Verma RK, M Sachan, K Vishwakarma, N Upadhyay, RK Mishra, DK Tripathi, S Sharma (2018). Role of PGPR in sustainable agriculture: molecular approach toward disease suppression and growth promotion. In: Role of Rhizospheric Microbes in Soil, pp: 259-290. Meena V (Ed). Springer, Singapore

Verma S (2016). A review study on Acacia tortilis. Intl J Life Sci Pharm Res 6:22-24

Wang B, YL Qiu (2006). Phylogenetic distribution and evolution of mycorrhizas in land plants. Mycorrhiza 16:299-363

Wu QS, RX Xia (2006). Effects of arbuscular mycorrhizal fungi on leaf solutes and root absorption areas of trifoliate orange seedlings under water stress conditions. Front Chin 3:312-317

Xiao D, R Che, X Liu, Y Tan, R Yang, W Zhang, X He, Z Xu, K Wang (2019). Arbuscular mycorrhizal fungi abundance was sensitive to nitrogen addition but diversity was sensitive to phosphorus addition in karst ecosystems. Biol Fert Soil 55:457-469 
Xiaoying C, S Fengbin, L Fulai, T Chunjie, L Shengqun, X Hongwen, Z Xiancan (2014). Effect of different arbuscular mycorrhizal fungi on growth and physiology of maize at ambient and low temperature regimes. Sci World J 5:1-7

Yadav A, VK Suri, A Kumar, AK Choudhary (2018). Effect of AM fungi and phosphorus fertilization on P-use efficiency, nutrient acquisition and root morphology in pea (Pisum sativum L.) in an acid alfisol. $J$ Plant Nutr 41:689-701

Ya-Dong S, H Xian-Chun, W Qiang-Sheng, Y Tian-Yuan, AK Srivastava, D Zhang, G Xiu-Bing, K Kuca (2021). Mycorrhizas promote P acquisition of tea plants through changes in root morphology and $\mathrm{P}$ transporter gene expression. S Afr J Bot 137:455-462

Yasser IE, AH Badreya, MA Eman (2021). Response of Nemesia (Nemesia $\times$ hybridus) plants to different irrigation water sources and arbuscular mycorrhizal fungi inoculation. Agric Water Manage 243:1-10
Yooyongwech S, N Phaukinsang, S Cha-Um, K Supaibulwatana (2013). Arbuscular mycorrhiza improved growth performance in Macadamia tetraphylla L. grown under water deficit-stress involves soluble sugar and proline accumulation. J Plant Growth Regul 69:285-293

Zaidi A, M Khan, M Ahemad, M Oves (2009). Plant growth promotion by phosphate solubilizing bacteria. Acta Microbiol Immunol Hung $56: 263-284$

Zhu XQ, CY Wang, H Chen, M Tang (2014). Effects of arbuscular mycorrhizal fungi on photosynthesis, carbon content, and calorific value of black locust seedlings. Photosynthetica 52:247-252

Zou YN, QS Wu, YM Huang, QD Ni, XH He (2013). Mycorrhizalmediated lower proline accumulation in Poncirus trifoliate under water deficit derives from the integration of inhibition of proline synthesis with increase of proline degradation. PLoS One 8; Article e80568 